\title{
OPTIMAL DISPATCH OF ANCILLARY SERVICES VIA SELF-ORGANIZING MIGRATION ALGORITHM
}

\author{
Jakub NOVÁK - Petr CHALUPA - Vladimír BOBÁL *
}

\begin{abstract}
Modern electric power systems are large-scale systems with a complex structure comprised of interconnected networks. The balance between the generation and consumption of electricity has to be maintained at any moment. Transmission System Operator (TSO) uses ancillary services for keeping the domestic power balance. Selection of the services can be viewed as an optimization problem. In this contribution the optimization problem is solved via the Self-Organizing Migration Algorithm which belongs to the class of evolutionary algorithms. The comparison with historical activations of ancillary services showed the improved performance in terms of reduced cost for ancillary services.
\end{abstract}

K e y w o r d s: evolutionary computations, ancillary services, frequency control, optimal dispatch

\section{INTRODUCTION}

Modern electric power systems are large-scale systems with a complex structure comprised of interconnected networks. The European Network of Transmission System Operators for Electricity (ENTSO-E) coordinates the operation and development of the electricity transmission grid in 34 countries, including Czech Republic as depicted in Fig. 1. Since 2009, the ENTSO-E is a successor of the Union for the Coordination of Transmission of Electricity (UCTE). ENTSO-E transmission system is divided into control areas - typically by countries, although some subsystems are joined together to create utilities that are able to operate independently in case of emergency. Transmission System Operator (TSO) manages the operation of the power system within the control area and coordinates its activities with neighbouring utilities. The TSO is responsible for taking care of the safe transmission of electricity, the reliability and stability of the system and balancing supply and demand at any time. Since the TSO does not own any generation, it must ensure that there is sufficient unloaded capacity among on-line generators for maintaining the reliability of the system. To reduce the risk, some ancillary services are obtained through long term contract and some are bought through spot market.

The provision of ancillary services is an economic and technical issue that must be addressed in all deregulated power market structures. Since TSO works on behalf the users of the system it has to decide how much ancillary services should be bought. If there are not enough services then TSO cannot ensure the security of the system. However, the costs for the AS are passed to system user. The interdependence between the security margin, the energy schedules and the ancillary services makes the problem of choosing the optimal energy and ancillary service (AS) configuration difficult. The problem is formulated as a mixed integer, nonlinear optimization problem taking into account both continuous and discrete control variables. The objective of the reserve dispatch in a multiarea electricity market is to minimize the expenses for the reserves subject to a number of constraints.

The dispatch problem for electricity generation has been the focus of numerous studies. Arnott et al [1] reviewed frequency control practices in different countries, summarised experience gained from market changes and suggested ways in which frequency control needs further development. An adaptive scheme which uses both frequency and rate-of-change of frequency measurements to set load shedding and technique for coordination with spinning reserve is presented in [2]. Wang et al in [3] modified P-Q decoupled optimal power flow (OPF) to employ for the real-time optimal dispatch problem.

The power system operator is faced to many optimization problems in power system operation. Many classical optimization techniques have been applied to these problems, such as linear or quadratic programming [4]. These classical techniques use approximations and linearization procedures in order to limit complexity of the optimization problem. In [5] a heuristic based technique called horizon scan is used to find optimal solution for the problem of economic dispatch with linear and nonlinear constraints given by the dynamics of power systems. Pareto-based evolution strategy was applied in [6] to minimize the costs for providing reserve aid for covering transmission losses. In [7] the optimal preventive control actions are obtained via multi-objective fuzzy linear programming (MFLP) technique. Other optimization techniques such as particle swarm optimization [8] or genetic algorithms [9] have been also applied for dispatch optimization.

\footnotetext{
* Department of Process Control, Faculty of Applied Informatics, Tomas Bata University in Zlín, Nám. T.G. Masaryka 5555,76001 Zlín, Czech Republic, jnovak@fai.utb.cz
} 


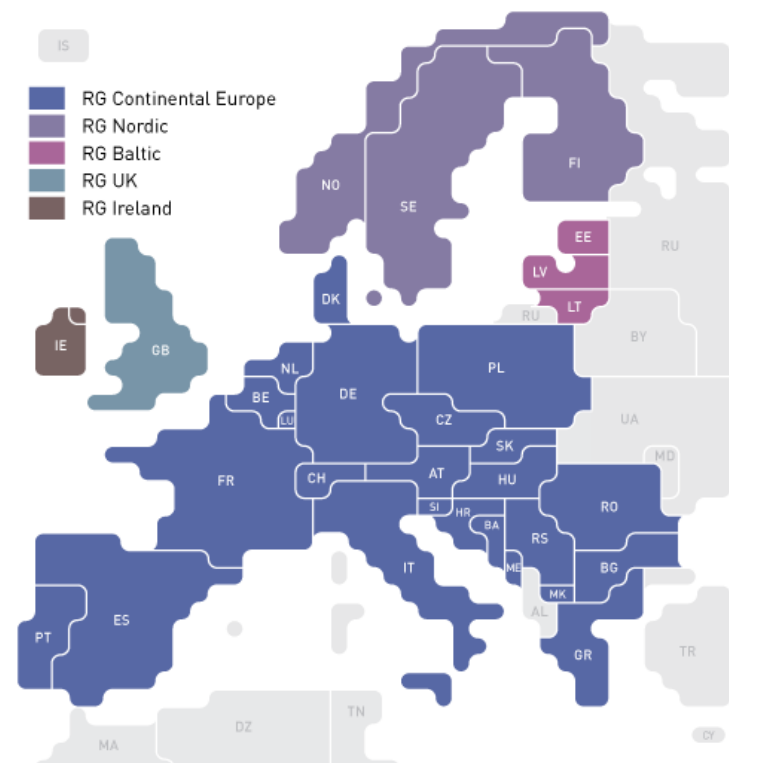

Fig. 1. ENTSO-E regions of synchronously interconnected systems

\section{ANCILLARY SERVICES}

Power systems are subjected to sudden and unpredictable changes due to changes of generation and fluctuations of loads. Therefore continuous regulation is essential in maintaining system frequency. If generation exceeds load then frequency rises. If load exceeds generation frequency falls. Continuous regulation is also important in controlling inter-area power flows. If generation exceeds load within one balancing area, then power will flow over the transmission line ties to adjacent areas. To be able to respond to contingencies without affecting overall reliability system operators have a coordinated set of operating reserves. If there is a generator outage the frequency has to return to its preset value of $50 \mathrm{~Hz}$ within 15 minutes. The Czech TSO operator ČEPS defined following ancillary services to balance contingencies.

The transmission system operator (TSO) uses different ancillary services to maintain stability and reliability of the power grid. The types of ancillary services are defined in the Grid code [10] issued by TSO. Main types of ancillary services are describes further in this chapter.

\subsection{Unit primary f control (PR)}

Unit primary frequency control is a local automatic function ensured by the primary regulation circuits. It is based on a precisely defined change in a generating unit's output dependent on the frequency deviation from its predefined value.

The primary control is activated automatically by each block that provides PR. Thus PR cannot be directly used by TSO.

\subsection{Unit secondary $P$ control (SR)}

Unit secondary $\mathrm{P}$ control (SR) is a process involving a regulated generating unit output value change as required by the secondary frequency regulator and the transferred power balance. The utilization of a regulation reserve of SR (hereinafter RZSR) is dependent upon the TSO dispatch centre secondary regulator algorithm.

The requests of SR change are sent from the TSO dispatch centre automatically according to current situation in the transmission system. Thus, course of the SR is considered as known but un uninfluenceable from the point of view of the optimization problem this paper is coping with.

\subsection{Unit tertiary spinning $P$ control (TR)}

Unit tertiary spinning $\mathrm{P}$ control consists of a change in unit output based on a request sent to the power plant by the ČEPS dispatch centre. The reserve marked RZTRis used for power decrease and that marked RZTR+ for power increase. The activation of tertiary spinning reserve is done strictly in rising-price manner. The positive tertiary spinning reserve is controlled by the Positive Critical Price Coefficient $(\mathrm{CPC}+)$ and similarly the negative tertiary spinning reserve is controlled by Negative Critical Price Coefficient (CPC-). The dispatch centre sends the value of $\mathrm{CPC}+$ to the terminals of all generating blocks that provide TR.

\subsection{Quick-start 10 minute reserve (QS)}

The quick start 10 minute reserve concerns units capable of offering the agreed reserve, the discontinuation of pumping (in the case of pumped storage hydro plant) or the discontinuation of pre-planned pumping within 10 minutes of a command from the TSO dispatch centre.

\subsection{Stand-by reserve (DZ)}

The stand-by reserve accessible within t-minutes concerns shut down, reserve-state generating units which, at the request of the TSO, are capable of guaranteeing synchronization and able to reach nominal or previously agreed power no later than within the agreed time. The stand-by reserve accessible within t-minutes is the terminal output of the relevant unit less self-consumption.

\subsection{Other ancillary services}

In addition to the previously mentioned ancillary services, there are other ancillary services, which are less important for the solved optimization problem. These services include:

- Load change - load decrease or increase by a predefined and guaranteed value of regulation reserve according to the request from the TSO.

- Generation shedding - units capable of decreasing power output by a reserve value agreed in advance, 


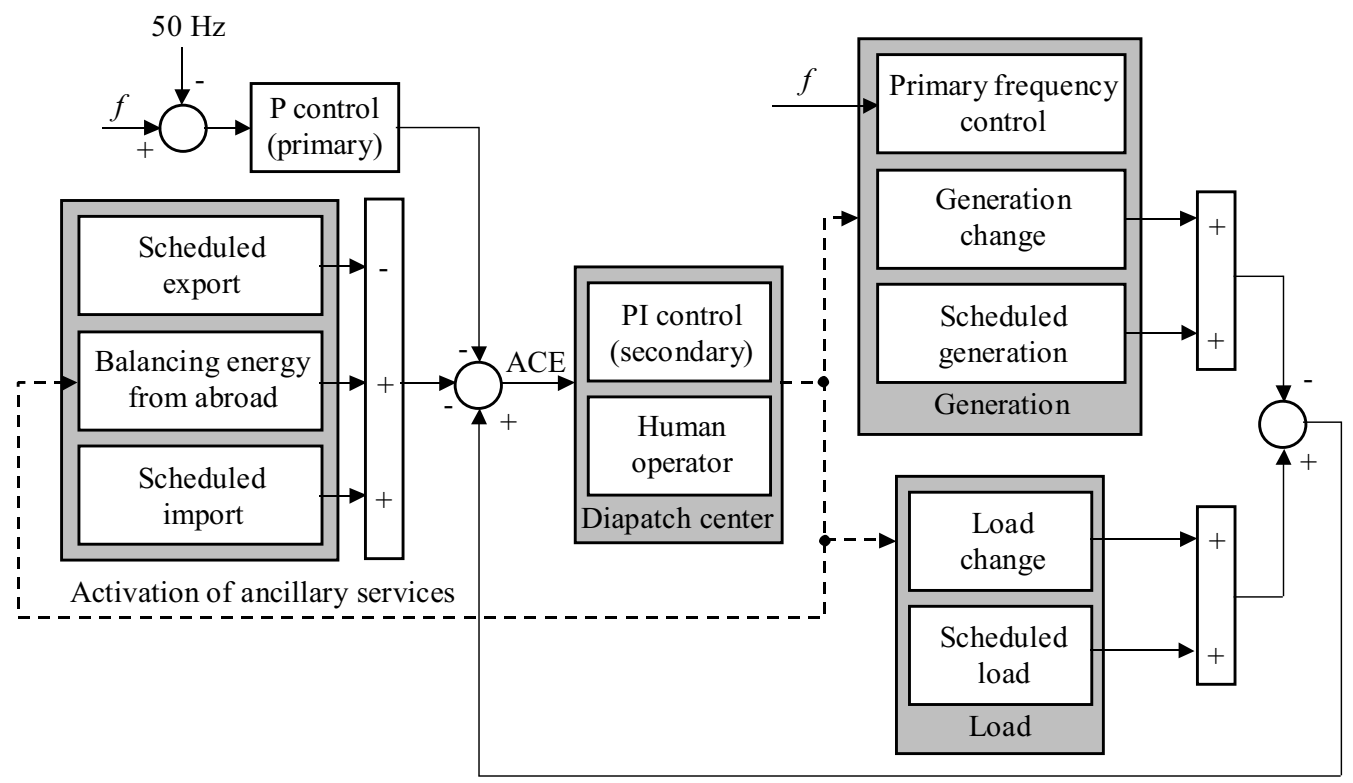

Fig. 2. Scheme of balance control in the transmission grid

or capable of being shut down completely or of not being started up within 30 minutes of receipt of an instruction from the TSO dispatch centre.

- Cross-border provision of non-guaranteed regulation energy.

The key point is to optimally select the appropriate AS set at lowest costs for AS reservation. Currently, an expert system, developed in Havel et al $[11,12]$, helps the Czech TSO to better understand the transmission system and recommends the optimal set of AS to be purchased to meet the required reliability indices. The expert system uses Monte-Carlo simulations for analysis of the behaviour of the grid under the different distribution of AS. The model also includes a model of a human operator at the dispatch centre who activates the AS.

\section{REAL TIME FREQUENCY CONTROL}

Frequency control is the real-time control of all generation and transmission resources that are currently online and available to meet load and to maintain reliability within the control area. Frequency will deviate from the nominal setting whenever there is any imbalance between generation and load as the imbalance will be drawn from the kinetic energy stored in the rotating masses of the generators. ACE is a difference between the scheduled and actual foreign power exchange corrected with the effect of the primary control, which acts independently of the central controller, to avoid counter-regulation. The ACE should be kept at the zero level since negative ACE represents unscheduled export and positive ACE represents unscheduled import.
The Area Control Error (ACE) is defined in ENTSO-E Operational Handbook [13] as

$$
\mathrm{ACE}=\Delta P+K \Delta f
$$

where $\Delta P(\mathrm{MW})$ is a power control error (measured difference between scheduled and actual interchange with neighbouring areas), $\Delta f(\mathrm{~Hz})$ is a frequency error (deviation of the measured frequency $f_{m}$ from the frequency set-point $\left.f_{S P}\right)$

$$
\Delta f=f_{m}-f_{S P}
$$

and $K(\mathrm{MW} / \mathrm{Hz})$ is used to link frequency and power deviations and it is called $\mathrm{K}$-factor in the Operational Handbook terminology. The term compensates for the action of the distributed primary control within the area in order to avoid counter-regulation from the central PI controller at the TSO's dispatch centre. This ensures that the secondary control will only be called up in the control area which is the source of the disturbance.

During real time, the TSO shall dispatch generating units, loads and system resources to procure imbalance energy as depicted in Fig. 2.

In addition, the TSO may also need to purchase additional AS if the services arranged in advance are used to provide imbalance energy, and such depletion needs to be recovered to meet reliability contingency requirements. TSO chooses the generating units, loads and system resources only based on price, and the effectiveness (location and ramp rate) of the resource concerned to respond to the fluctuation in generation or demand.

Dispatch centre uses a database for dispatch of ancillary services that includes:

- generating unit name,

- quantity bid,

- price, 


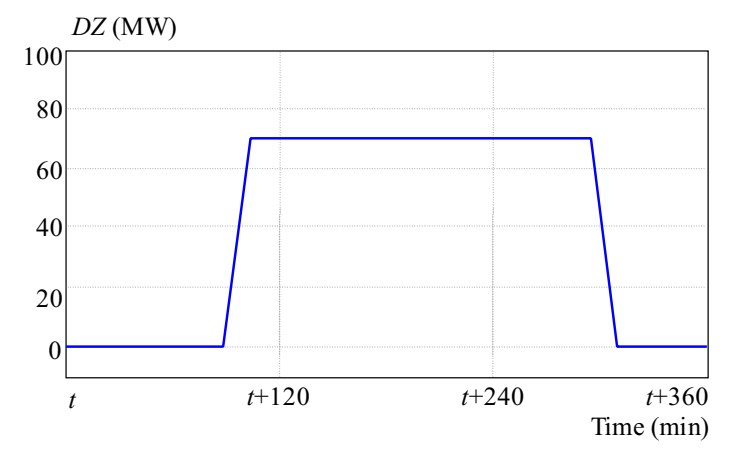

Fig. 3. Activation of Stand-by reserve

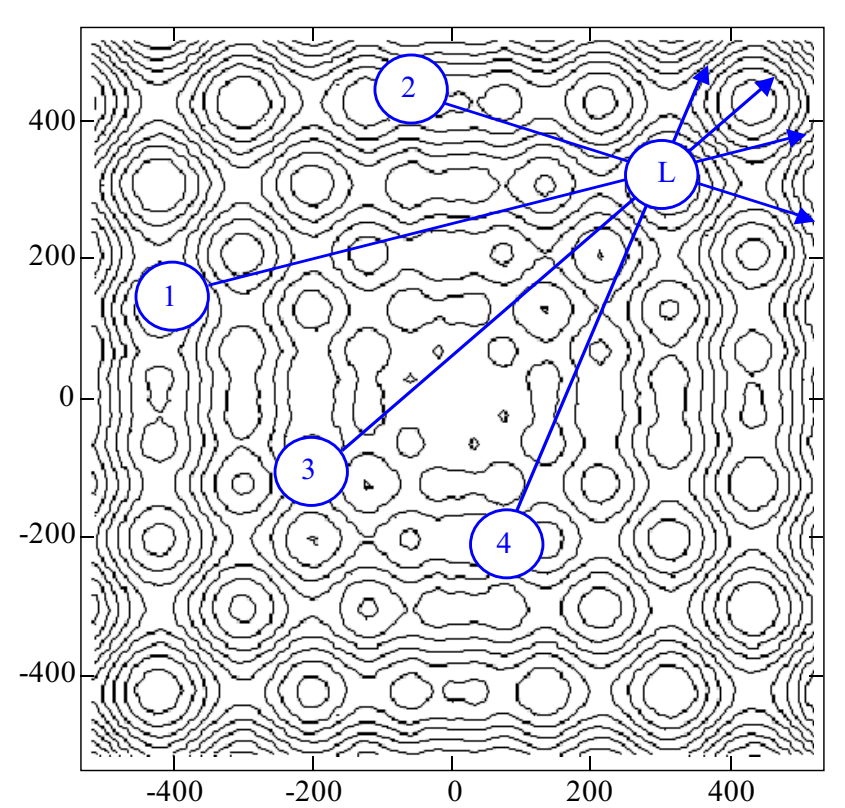

Fig. 4. Movement of individuals in a 2-dimensional optimization problem (L-leader)

- type of ancillary service provided.

The primary control loop is the first to react for imbalances. In order to maintain system frequency at the nominal value and schedules between control areas, a secondary frequency control loop or Automatic Generation Control (AGC) coordinates the individual raise and lower signals to the generators. The control centre gathers the relevant frequency and power flow information and sends the appropriate set point adjustments for units that can provide ancillary services. The first step of the dispatch optimization process is forecasting the ACE for the prediction horizon of 6 hours. Using the statistical data from past years, actual and past ACE, daily diagrams and exponential forgetting, a vector that contains 5-minutes prediction of ACE is constructed.

The scheduled unit activation that would provide the power balance control with minimal costs is computed using optimality criterion

$$
J=J_{\text {energy }}+J_{\text {control }}
$$

where $J_{\text {energy }}$ is the cost for energy supplied by all the units providing ancillary services

$$
J_{\text {energy }}=J_{\text {energy }}^{\mathrm{SR}}+J_{\text {energy }}^{\mathrm{TR}}+J_{\text {energy }}^{\mathrm{QS}}+J_{\mathrm{energy}}^{\mathrm{DZ}}+J_{\mathrm{energy}}^{\mathrm{HV}}
$$

and $J_{\text {control }}$ is control performance penalization. The energy costs for stand-by reserve are given as

$$
J_{\text {energy }}^{\mathrm{DZ}}=\sum_{k=1}^{360} \sum_{i=1}^{N^{\mathrm{DZ}}}\left(C_{i}^{\mathrm{DZ}}(k) P_{i}^{\mathrm{DZ}}(k) T(k)+C_{i}^{\mathrm{SU}}(k)\right)
$$

where $N^{\mathrm{DZ}}$ is the number of units providing stand-by reserve, $C_{i}^{\mathrm{DZ}}$ is the price of the unit, $P_{i}^{\mathrm{DZ}}$ is the output of the unit $i$ at the sample point $k, T(k)$ is the sampling interval and equals to 1 minute and $C_{i}^{\mathrm{SU}}$ are the startup cost of unit $i$ at time $k$. The energy costs for other ancillary services are computed in the same fashion. The control performance part $J_{\text {control }}$ of the criterion (3) ensures the minimization of ACE as it penalizes every MWh of uncompensated closed-loop ACE

$$
J_{\text {control }}=\sum_{k=1}^{360} C_{\mathrm{ACE}}(k)\left|P(k)-\mathrm{ACE}_{0}(k)\right| T(k)
$$

where the term inside absolute value is the closed-loop $\mathrm{ACE}$ at sample time $k, \mathrm{ACE}_{0}$ denotes the open-loop ACE predictions and $C_{\mathrm{ACE}}$ is the penalization price of uncompensated ACE. The output of all units $P$ at each sample time is defined as sum of outputs of all units

$$
\begin{aligned}
P(k)=\sum_{i=1}^{N^{\mathrm{QS}}} P_{i}^{\mathrm{QS}}(k)+\sum_{i=1}^{N^{\mathrm{TR}}} P_{i}^{\mathrm{TR}}(k)+ & \\
& \sum_{i=1}^{N^{\mathrm{DZ}}} P_{i}^{\mathrm{DZ}}(k)+P^{\mathrm{HV}}(k)+P^{\mathrm{SR}}(k) .
\end{aligned}
$$

The optimization task is to find the minimum of the overall costs with respect to constraints. The main constraints for the optimized parameter arise from the amount of ancillary services that have been purchased in a day-ahead market or through bilateral contract. The amount of energy that can be purchased from abroad is limited by the free capacity on profiles between the national power grids. Other constraints for parameters that describe the course of AS on the future horizon are time delays, rates of growth, maximal and minimal amount of activations of each AS. E.g. Stand by reserve which can be activated only at its nominal value has time delay of 90 minutes. It reaches the nominal value in 15 minutes and must be activated for minimal time of 2 hours. The activation of the block that provides Stand-by reserve with the nominal value of $70 \mathrm{MW}$ for 3 hours at the current time instant $t$ would result in the power output as depicted in Fig. 3. 


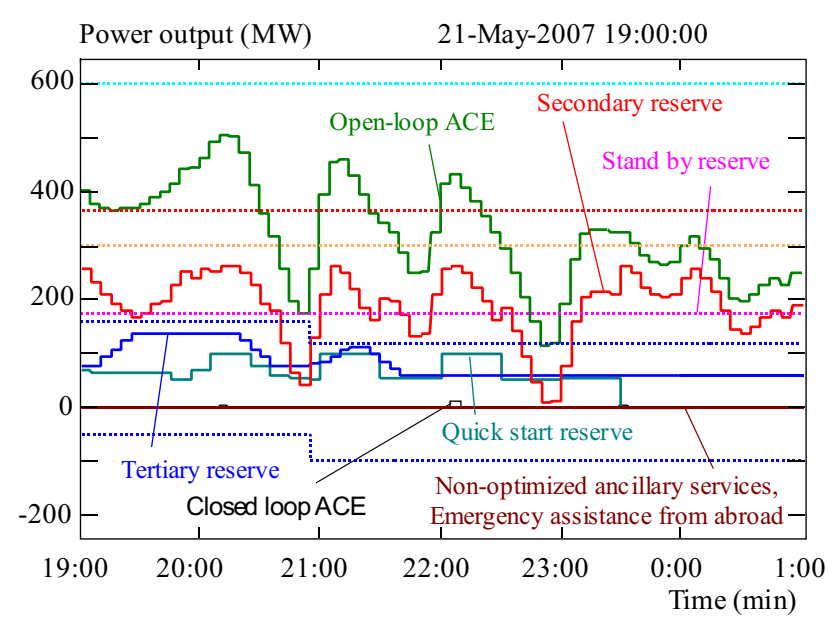

Fig. 5. Optimal schedule of ancillary services at time 19:00

\section{OPTIMIZATION TOOL}

Self-Organizing Migration Algorithm [14] is modelled on the social behaviour of co-operating individuals and has been proven that the algorithm has the ability to converge towards the global optimum. Each individual represents a single solution to a problem. These individuals are mutually influenced during the search process, which leads to forming/cancelling groups of individuals. Such groups organize themselves the movement of individuals, therefore the adjective self-organizing. The main benefit of this algorithm is its ability to process diverse data types of parameters like integers, real or discrete values. After the initialization where managing and finishing parameters are defined, the population of individuals is created. Each individual is evaluated by a fitness function and given the value of this function. The individual with the minimal costs is chosen as a leader in next migration cycle. Then all individuals start to move to the leader in steps. After each steps the individuals are again evaluated by the cost function. If the value of evaluation is lower than the previous one it is remembered. After each individual reaches the last step it returns to a new position on his migration trajectory where the best fitness was found, i.e. beside the leader all the individuals will be moved to a new location. In other words they did searching in the state space and migrated. Thus a single cycle has been finished and new one will immediately start after a new leader (the best individual) is determined.

The movement of an individual can be described by Equation (8).

$x_{i, j}^{M K+1}=x_{i, j, \mathrm{START}}^{M K}+\left(x_{L, j}^{M K}-x_{i, j, \mathrm{START}}^{M K}\right) * t *$ PRTVector

where $x_{i, j}^{M K+1}$ is value of $i$-individual of $j$-parameter in step $t$ in next migration step $M K+1, x_{i, j}^{M K}$ is value of $i$-individual of $j$-parameter in step $t$ in actual migration step $M K, x_{L, j}^{M K}$ is value of Leaders $j$-parameter in step $t$ in actual migration step $M K$.
Parameter $t$ takes values from 0 to 1 by a defined step. SOMA uses a parameter called PRT to achieve perturbation. This parameter has the same effect for SOMA as mutation has for genetic algorithms. It is defined in the range $[0,1]$ and is used to create a perturbation vector (PRTVector). If an element of the perturbation vector is set to zero, then the individual is not allowed to change its position in the corresponding dimension. The movement of the individuals is shown in Fig. 4. The size of population remains the same, even the individuals are the same. The acquired knowledge remains in each individual and we can observe certain learning process during migration cycles. The only kind of selection can be observed in choosing the leader, which depends on the quality of an individual. For optimization of ancillary services dispatch a single individual represents a single solution of the dispatch problem $i e$ time series of activated AS.

\section{SHORT TERM PERFORMANCE EVALUATION}

To test the optimization properties of the SOMA algorithm the proposed algorithm was applied to 2 case studies. At each sampling interval, the TSO control centre had the following information

- Prediction of ACE for the next 6 hours.

- Prices and sizes of blocks that can provide ancillary services for the next 6 hours.

The database of ancillary services that can be used for dispatch was described in Section 2. If two generating units provide the same ancillary service and have the same price they are replaced by a dummy source with size given by the sum of capacities of both sources to reduce the number of parameters.

The following parameter values to guide its search: population size of 100 and 100 migrations. The optimization time depends not on the number of parameters but on the computation time of the cost function. Depending on the step of the SOMA algorithm 100-500 computations of the cost function are needed for each generation.

At each sampling point the SOMA optimization algorithm is used to find the cost-optimal solution of the problem defined by cost function (4). This solution is given in terms of a time series of activated AS for next 6 hours.

The optimized schedule with all the ancillary services is shown in Fig. 5. The open-loop ACE prediction (green line) is compensated using Quick-start reserve, Tertiary reserve and Secondary reserve. The closed-loop ACE is reduced to zero or close to zero. As in the predictive control strategy only the set-point changes to generating blocks that refers to the current sampling point are applied. The computation time for a single optimization is 15 seconds on Intel Pentium 3.0 GHz, 4 GB RAM. The penalization price of uncompensated ACE was set to 500 EUR/MWh. To compare the automatic controller with a human operator the historical data from the TSO were 
Table 1. Regulation energy utilization and costs for Test I

\begin{tabular}{lcccccccccc}
\hline & SR+ & SR- & TR+ & TR- & SbR & QS & EA & Other & ACE & AS costs \\
\hline historical activation & 47.3 & 20.4 & 21.3 & 0 & 10.9 & 0 & 0 & 0 & 100 & 100 \\
automatic controller & 58.1 & 19.7 & 11.7 & 1.9 & 0 & 8.5 & 0 & 0 & 91 & 81 \\
\hline
\end{tabular}
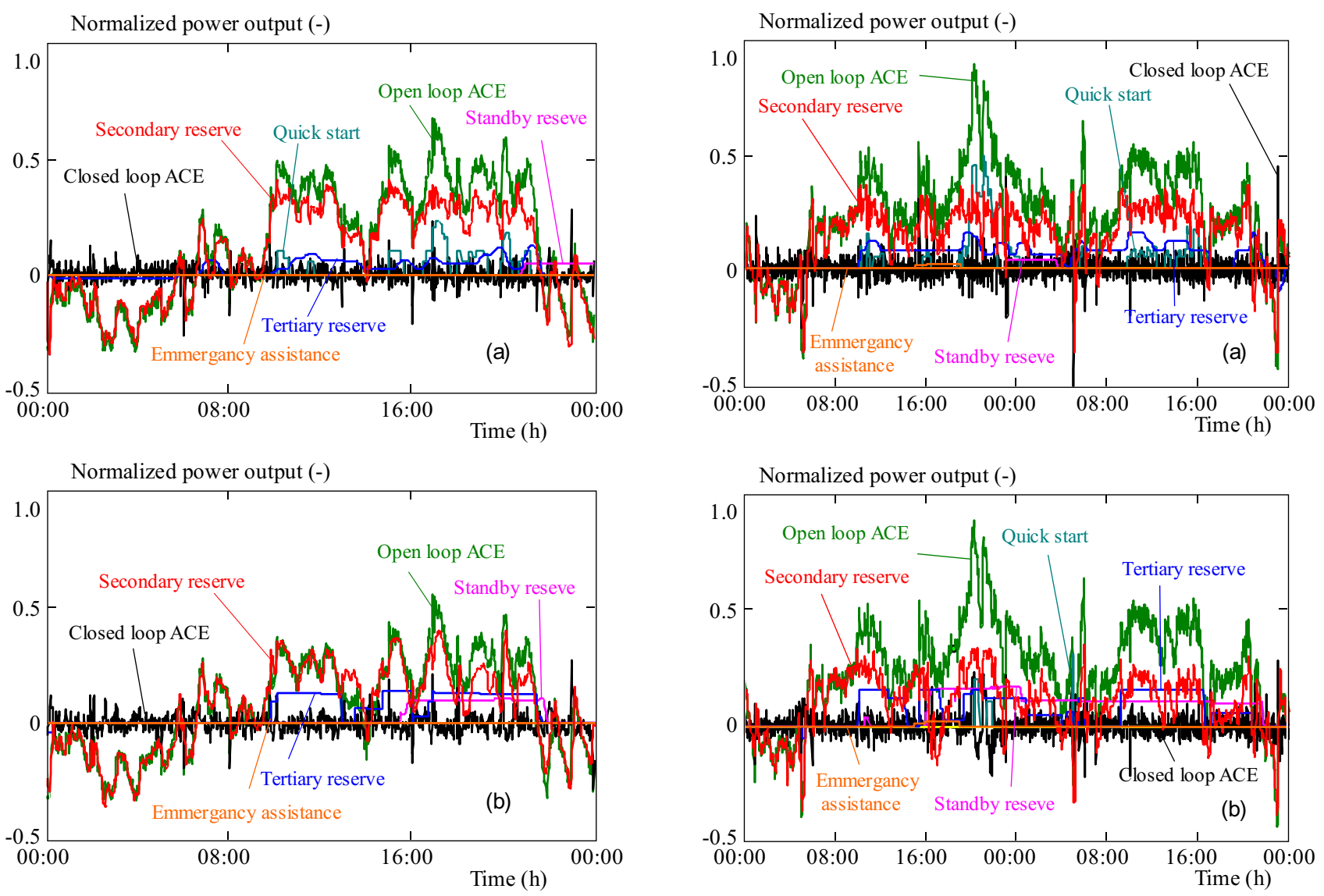

Fig. 6. Short term test I: (a) - automatic controller, (b) - historical activation

Fig. 7. Short term test II: : (a) - automatic controller, (b) historical activation

Table 2. Regulation energy utilization and costs for Test II

\begin{tabular}{lcccccccccc}
\hline & SR+ & SR- & TR+ & TR- & SbR & QS & EA & Other & ACE & AS costs \\
\hline historical activation & 40.1 & 7.3 & 23.7 & 0 & 26.1 & 1.4 & 0 & 0.5 & 100 & 100 \\
automatic controller & 57.7 & 6.0 & 22.4 & 0.4 & 1.3 & 11.7 & 0 & 0.5 & 90 & 67 \\
\hline
\end{tabular}

used for evaluation of the automatic controller. The number of units providing ancillary services varies from 15 to 64 depending on the day and hour.

The superiority of the proposed automatic controller is shown on two short term tests. For each test a figure comparing the historical activation with the optimized schedule and a table showing the relative regulation energy utilization, costs for regulation energy and uncompensated ACE is presented. The first short term test (Fig. 6) presents a performance of the automatic controller during generation unit outage that last 12 hours. Units that provide tertiary spinning reserve units and with price lower than the price of Secondary control are activated. The ac- tivation is provided by manipulating the value of positive $\mathrm{CPC}+$. The power imbalance is further controlled by activating the units that provide Quick start reserve with low price. The comparison shows the improved performance where the energy costs were reduced by approximately $19 \%$ with closed-loop ACE reduced by $9 \%$. The automatic controller applied less Stand-by reserve and more Quick-start reserve in the simulation. The distribution of regulation energy among the regulation reserves in percents is presented in Tab. 1. The column "Other services" is for services that were not included in optimization.

The second short term test (Fig. 7) presents different situation with long-lasting lack of power in the system. 
In the second test the energy costs were reduced by approximately $33 \%$ with closed-loop ACE being reduced by $10 \%$. The simulation results are presented Tab. 2 .

The automatic controller is most beneficial in terms of cost reduction in situations when secondary frequency control is insufficient to compensate the open-loop ACE alone and activation of other ancillary services is necessary as can be seen in short-term tests. The optimization over the prediction horizon also reduced the amount of counter-regulation when both positive and negative ancillary services are activated at the same time.

\section{CONCLUSIONS}

The paper proposed an approach to dispatch of ancillary services using the Self-Organizing Migration Algorithm (SOMA). The short term tests confirmed that the regulation energy cost can be reduced without compromising the control performance by application of the automatic controller that optimizes the activation of ancillary services on the 6-hour horizon. SOMA is capable of successfully finding the optimal activation schedule with minimal costs even though the optimization problem contains several local minima with continuous, discrete and binary parameters.

The results of proposed automatic controller can be compared with the activation schedule produced by human operators and the differences can be used for training of operators or as an assistance tool for power balance control. Future research will be focused on investigation of the performance of the automatic controller in long terms (eg one year).

This work was supported by the Ministry of Education of the Czech Republic under the grants 1M0567.

\section{REFERENCES}

[1] ARNOTT, I.-CHOWN, G.-LINDSTROM, K.-POWER, M.-BOSE, A.-GJERDE, O.-MORFILL, R.-SINGH, N. Frequency Control Practices in Market Environments, Proceedings of the Quality and Security of Electrical Power Delivery systems, $143-148$.

[2] ChuvyChin, V. N.-GUROV, N. S.-VEnKATA, S. S.BROWN, R. E. : An Adaptive Approach to Load Shedding and Spinning Reserve Control during under Frequency Conditions, IEEE Transactions on Power Systems 11 (1996), 1805-1810.

[3] WANG, X.-SONG, Y. H.-LU, Q. : A Coordinated Real Time Optimal Dispatch Method for Unbundled Electricity Markets, IEEE Transactions on Power Systems 17 (2002), 482-490.

[4] FAN, J. Y.-ZHANG, L.: Real-Time Economic Dispatch with Line Flow and Emission Constraints Using Quadratic Programming, IEEE Transaction on Power Systems 13 (1998), 320-325.

[5] CHAND, P.-SUGIANTO, L. F. : Co-Optimization of Energy Market and Ancillary Services, in: Power Engineering Conference, 2005, pp. 1001-1006.

[6] SCHMitT, A.-VERSTEGE, J. F.: A Multi-Criteria Optimization of Ancillary Services in a Competitive Energy Market, in: IEEE Power Engineering Society Winter Meeting, 3, 2001, pp. 1061-1066.
[7] ABOU El-ElaA, A. A.-BISHRA, M. A.-ALlama, S. M.El-SEHIEMY, R. A.: An Emergency Power System Control Based on the Multi-Stage Fuzzy Based Procedure, Electric Power Systems Research 77 (2007), 421-429.

[8] YOSHIDA, H.-KAWATA, K.-FUKUYAMA, Y.-TAKAYAMA, S.-NAKANISHI, Y.: A Particle Swarm Optimization for Reactive Power and Voltage Control Considering Voltage Security Assessment, IEEE Transactions on Power Systems 15 (2000), 1232-1239.

[9] DAMOUSIS, I. G.-BAKIRTZIS, A. G.-DOKOPOULOS, P. S. : Network-Constrained Economic Dispatch Using Real-Coded Genetic Algorithm, IEEE Transactions on Power Systems 18 (2002), 198-205.

[10] Grid Code. ČEPS, Czech Republic. 2006. Available on-line: http://www.ceps.cz/doc/kodex/part_i_ii_ii__an_fin_rev07.pdf.

[11] HAVEL, P.-HORÁČEK, P.-FANTÍK, J.-JANEČEK, E. : Criteria for Evaluation of Power Balance Control Performance in UCTE Transmission Grid, in: 17th IFAC World Congress, 2008, pp. 6998-7003.

[12] HAVEL, P.—HORÁČEK, P.—ČERNÝ, V.-FANTÍK, J. : Optimal Planning of Ancillary Services for Reliable Power Balance Control, IEEE Transactions on Power Systems 23 (2008), 1375-1382.

[13] P1 Policy 1: Load-Frequency Control and Performance [C] v3.0 rev 15/01.04.2009, European Network of Transmission System Operators for Electricity. 2009. Available online: https://www.entsoe.eu/fileadmin/user_upload/_library/ publications/entsoe/Operation_Handbook/Policy1_final.pdf..

[14] BABU, B. V.-ONWUbOLU, G.: New Optimization Techniques in Engineering, Springer-Verlag, UK, 2004.

Received 18 April 2011

Jakub NOVÁK (Ing, PhD) was born in Zlín, Czech Republic. He received his MSc. Degree in 2002 from the Tomas Bata University in Zlín. He received his PhD degree in Technical Cybernetics at Tomas Bata University in Zlín in 2007. He works as a researcher at Centre for Applied Cybernetics at the same university. Current work covers following areas: modelling electricity networks, multiple model control schemes, predictive control. He is an author or co-author of over 20 publications in journals and conference proceedings.

Vladimír BOBÁL (Prof, Ing, CSc) graduated from the Faculty of Mechanical Engineering, Brno University of Technology, Czech Republic in 1966. He received his CSc. degree in Technical Cybernetics at Institute of Technical Cybernetics, Slovak Academy of Sciences in Bratislava, Slovak Republic. From 1969 he has been working as a senior lecturer, associate Professor and now as Professor in the Department of Process Control, Faculty of Applied Informatics of the Tomas Bata University in Zlín, Czech Republic. His research interests are adaptive control systems, predictive control and system identification. He is author or co-author of and over 270 contributions in scientific journals and conference proceedings.

Petr CHALUPA (Ing, PhD) was born in Zlín, Czech Republic in 1976. He graduated from Brno University of Technology in 1999. He obtained his PhD in Technical Cybernetics at Tomas Bata University in Zlín in 2003. He works as a researcher at Centre for Applied Cybernetics at Tomas Bata University in Zlín. His research interests are adaptive and predictive control of real-time systems. He is author or co-author 1 scientific books and of over 70 contributions in scientific journals and conference proceedings. 